\title{
Clinical evaluation of the histochemical diagnosis of Hirschsprung's disease
}

\author{
L C BARR, J BOOTH, M I FILIPE, AND J O N LAWSON \\ From the Westminster Children's Hospital, London, and Guy's Hospital, London
}

SUMmaRY The histochemical staining of suction rectal biopsies for acetylcholinesterase (AChE) activity is a safe and accurate diagnostic tool in the management of patients with symptoms and signs of Hirschsprung's disease. In this study 101 patients who have undergone AChE analysis of suction rectal biopsies are presented in order to correlate histochemical findings with subsequent clinical course after a minimum of 18 months follow up. There were no false positive results, but the limitations and problems associated with the technique which we have encountered are discussed. Equivocal, atypical, or negative results when contradicting clinical features are indications for repeat biopsy or full thickness biopsy, and awareness of the situations in which false negative results may occur is essential.

The diagnosis of Hirschsprung's disease in patients presenting with symptoms and signs suggestive of this or related diseases is usually made on the basis of radiological investigation, anorectal manometry, and rectal biopsies.

The radiological features, of megacolon with cone shaped transition to the aganglionic segment, cannot be relied upon alone for diagnosis because they often do not develop until a number of weeks after birth, and do not always distinguish Hirschsprung's disease from other causes of megacolon. ${ }^{1}$

Anorectal manometry is a useful adjunct in the diagnosis of Hirschsprung's disease, as absence of the reflex relaxation of the internal sphincter is characteristic. There may be difficulties in gaining the child's cooperation, however, and in interpretation of the results, and rectal biopsy is still essential even when manometry is characteristic of Hirschsprung's disease. Manometry is particularly valuable in the diagnosis of ultrashort segment Hirschsprung's disease, a condition affecting essentially only the internal sphincter in which rectal biopsies may be normal. ${ }^{2-5}$

The classical method of rectal biopsy as described by Swenson involves taking a deep biopsy including the circular and longitudinal muscle laters, and involves a general anaesthetic and suturing of the biopsy site. This technique can be hazardous, and is

Address for correspondence: L C Barr. FRCS. Academic Surgical Unit, Royal Marsden Hospital, Fulham Road, London SW3 6JJ.

Received for publication 1 June 1984 unsuitable as a screening investigation. In addition it may make subsequent dissection of the rectum more difficult, should colonic resection be required. ${ }^{46}$

The development in recent years of histochemical staining techniques using superficial suction rectal biopsies of mucosa and submucosa for the diagnosis of Hirschsprung's disease represents a considerable advance particularly in the investigation of the neonate. Suction biopsies are easy to obtain, usually do not require anaesthesia, and have few complications. ${ }^{1}$

Staining suction rectal biopsies for acetylcholinesterase (AChE) activity reveals prominent nerve fibres in the lamina propria and muscularis mucosae in association with absence of ganglion cells in Hirschsprung's disease, and several studies have shown that these histochemical features correlate well with haematoxylin and eosin features, in which the diagnosis depends on the tedious examination of multiple sections to exclude the presence of any ganglion cells. ${ }^{7-9}$

The purpose of this paper is to correlate the histochemical features of rectal biopsies in a series of 101 patients with their final diagnosis and clinical outcome, and to outline some of the problems we have encountered during our seven years of clinical experience with the technique.

\section{Methods}

PATIENTS

One hundred and one patients undergoing $\mathrm{AChE}$ 
histochemical analysis of suction rectal biopsies at the Westminster Children's Hospital between 1975 and 1982 have been followed up for a minimum of 18 months, and have been reviewed to correlate histochemical diagnosis with final diagnosis and outcome.

Eighty one presented with chronic constipation, and 30 of these had symptoms dating from the first few weeks of life. Six of the 12 patients found to have Hirschsprung's disease in the 'chronic constipation' group had symptoms dating from birth. Fourteen presented with intestinal obstruction, eight during the neonatal period. Six patients presented with necrotising enterocolitis; four as neonates, one at age 8 months and one at 4 years, the latter two patients having typical clinical features of advanced untreated Hirschsprung's disease.

Suction rectal biopsies were taken at two centimetres above the mucocutaneous junction and at one centimetre intervals proximally, the number of biopsies depending on the age of the child and the suspected length of involved segment. The biopsies, consisting of mucosa and submucosa, were orientated before freezing in liquid nitrogen, and $10 \mu$ cyrostat sections were made. The histochemical technique was a modification of that described by Karnovsky and Roots, which stains cholinergic nerves an intense brown colour, making their identification easy. ${ }^{10}$

\section{Results}

Nineteen patients had the characteristic histochemical features of Hirschsprung's disease (Figs. 1 and 2). Subsequent routine pathological examination of surgical specimens, either of resected colon or of full thickness rectal biopsies at extended internal sphincterotomy, confirmed the diagnosis in all 19 cases (Table 1 ).

One patient had the characteristic features of neuronal colonic dysplasia as described by Meier Ruge and coworkers, with prominent ganglion cells and nerve trunks in the submucosa associated with increased AChE fibres in the lamina propria. ${ }^{111}$ Subsequent full thickness biopsies and colonic

Table 1 Histochemical findings

\begin{tabular}{lr}
\hline Hirschsprung's disease & 19 \\
Neuronal colonic dysplasia & 1 \\
Equivocal & 2 \\
Atypical & 4 \\
Normal & 75 \\
Total & 101 \\
\hline
\end{tabular}

resection confirmed the diagnosis. One patient with Hirschsprung's disease had some associated histological features of neuronal colonic dysplasia, and responded well to left hemicolectomy.

Two patients had equivocal biopsies with increased AChE fibres in the lamina propria and muscularis mucosae, not sufficient to be diagnostic of Hirschsprung's disease (Figs. 3a, and b). A full thickness biopsy at extended internal sphincterotomy in one showed no evidence of Hirschsprung's disease, and the other patient remains asymptomatic two years after one anal dilatation without repeat biopsy.

Four patients had atypical features, but which were not suggestive of Hirschsprung's disease (Figs. 4 and 5). All four had repeat biopsies; one had ulcerative colitis; one, who had presented with a perforated caecum at 2 years of age had a normal repeat biopsy and remains well without colonic resection; one, a neonate with intestinal obstruction, remains well with no surgical treatment; and one was found to have Hirschsprung's disease. This last patient, a 'false negative', was a male infant whose first biopsy had been peformed at 4 days of age.

Seventy five patients had normal histochemical features, and have been followed up for at least 18 months. Of the 66 patients in the "chronic constipation' group who had normal histochemistry, 27 had subsequent full thickness biopsies and extended sphincterotomies, and all biopsies were normal. The remaining 39 patients were successfully treated as shown in Table 2; two had anorectal studies suggestive of ultrashort segment Hirschsprung's disease and responded well to anal dilatation alone.

All four patients in the 'necrotising enterocolitis' group with normal histochemistry remained well with no clinical features of Hirschsprung's disease. These four patients were neonates in whom rectal biopsies were performed either before closure of colostomy (two patients) or because of persisting bowel dysfunction (two patients).

Of the five patients in the 'intestinal obstruction' group with normal histochemistry, two had 'false negative' results. One was a male neonate whose first biopsy was undertaken within the first week of life, but whose repeat biopsy subsequently confirmed the clinical suspicion of Hirschsprung's disease. The other was a case of total colonic aganglionosis who underwent total colectomy.

There were thus a total of three 'false negative' suction rectal biopsies, occurring in clinical situations in which other authors have also experienced false negatives. ${ }^{12} 13$ 


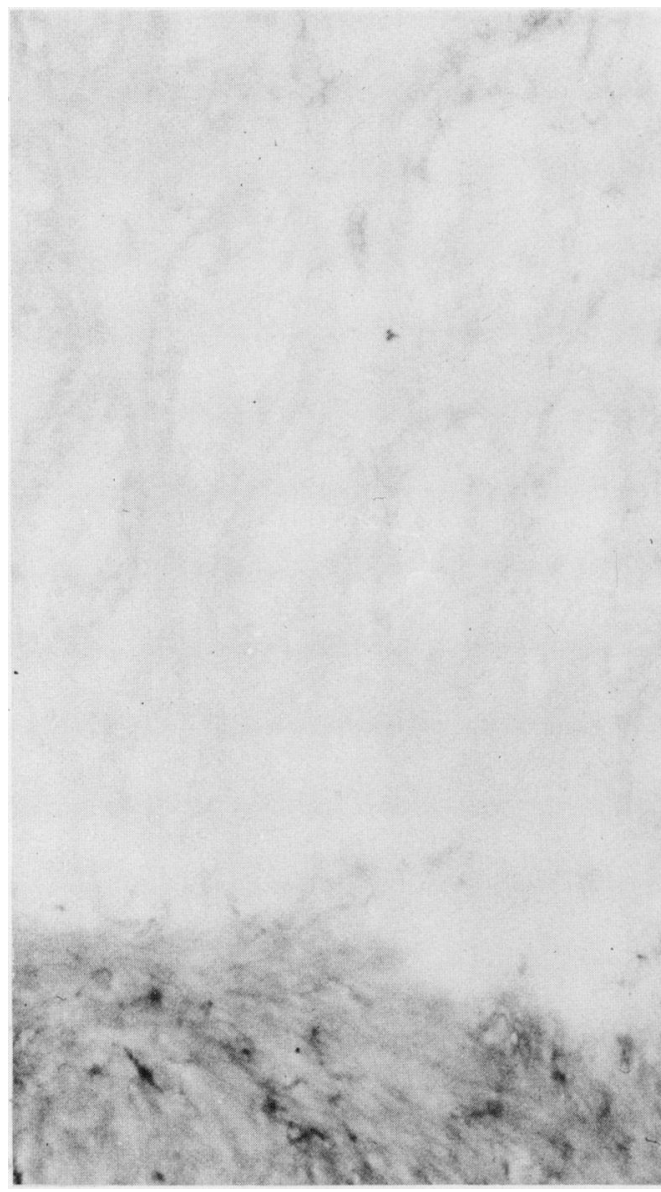

Fig. 1

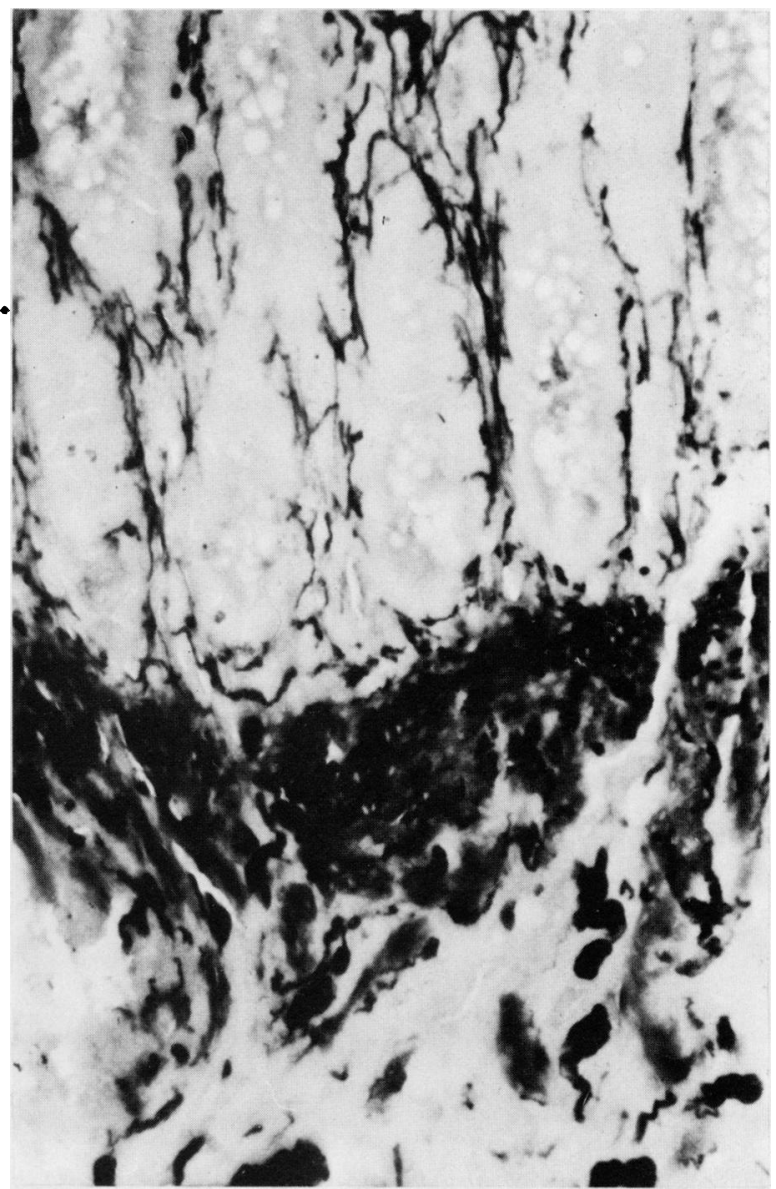

Fig. 2

Fig. 1 Normal rectal mucosa stained with the acetylcholinesterase (AChE) method. AChE positive parasympathetic nerve fibres are absent from both the lamina propria and the muscularis mucosae. $(\times 200$ original magnification).

Fig. 2 Hirschsprung's disease. Rectal mucosa shows an increased number of thick parasympathetic nerve fibres in the lamina propria and muscularis mucosae. Thick nerve trunks are also seen in the submucosa. $(\times 200$ original magnification, AChE method).

\section{Discussion}

The AChE histochemical staining technique on suction rectal biopsies has in our experience proved to be an accurate diagnostic tool, and a reliable screening test in patients with symptoms and signs of Hirschsprung's disease. Suction rectal biopsies are safer and easier to obtain than full thickness biopsies and, provided attention is paid to the technique in obtaining biopsies of mucosa and submucosa from appropriate levels with prompt orientation and freezing, a definitive diagnosis can be made with confidence on a relatively small biopsy. The histochemical technique allows the cholinergic fibres and ganglion cells to be easily identified, and is a less tedious method than conventional staining in which the diagnosis of Hirschsprung's disease depends on excluding the presence of any ganglion cells in multiple sections. There were no false positive results in our series, a finding which correlates with other authors' experiences. In addition, the technique allows the diagnosis of the related condition neuronal colonic dysplasia and possibly of other allied ganglion disorders, although a full thickness 

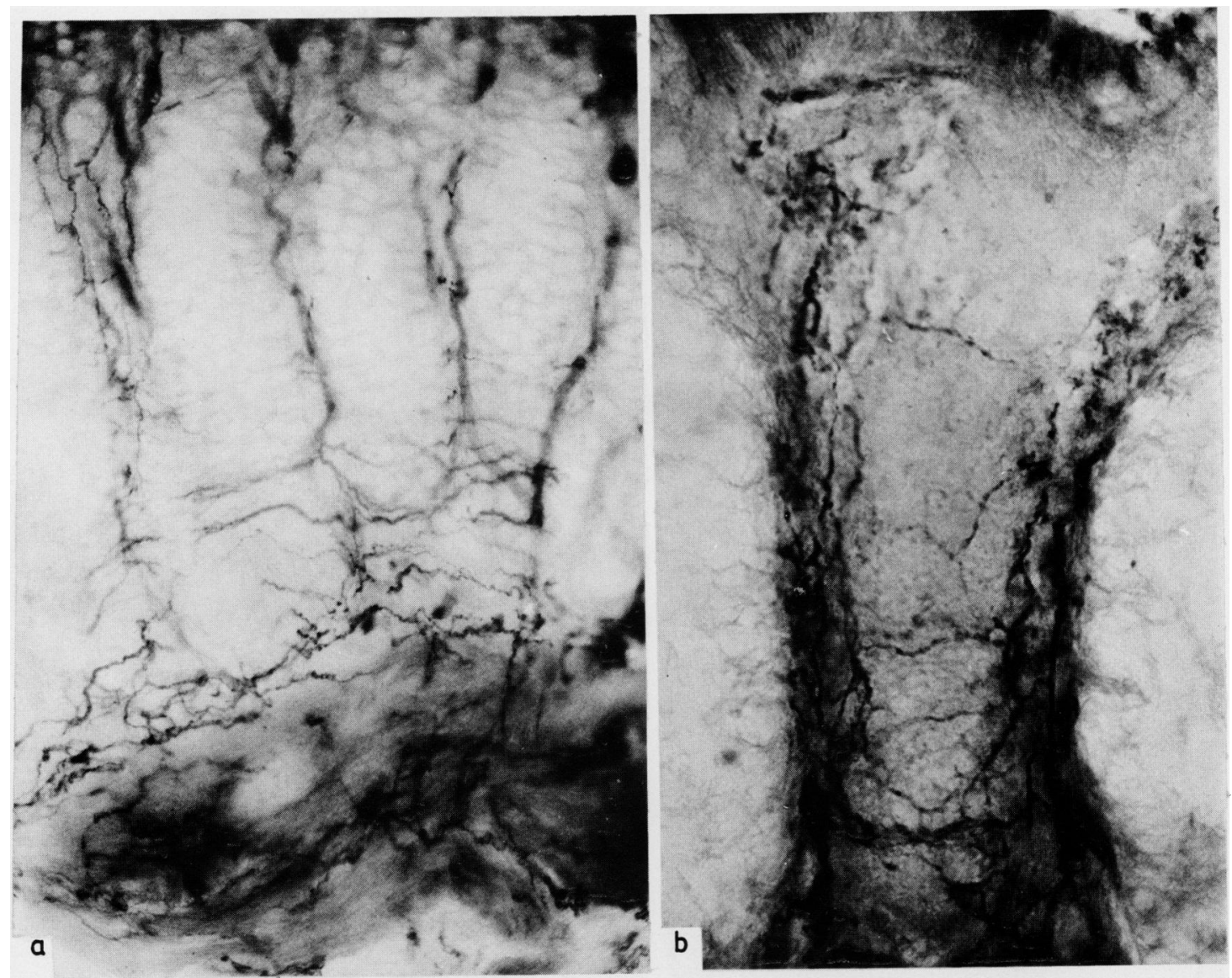

Figs. 3a, b Equivocal histochemical features. Rectal mucosa shows a network of acetylcholinesterase positive nerve fibres in both the lamina propria and muscularis mucosae. These features, although not fulfilling the diagnostic criteria, were suggestive of Hirschsprung's disease and therefore reported as 'equivocal'. Both these patients remain well on follow up with no large bowel symptoms; one following an extended sphincterotomy with normal conventional histology. $(a \times 200, b \times 500$ original magnification. AChE method).

biopsy should also be obtained for definitive diagnosis.

There are, however, a number of problems with the technique. AChE staining is only suitable for the left colon and rectum, as the activity of the extramural parasympathetic system decreases proximally from the anal canal so that its activity approaches zero at the splenic flexure. ${ }^{12}$ This means that histochemical staining of biopsies taken from the colon proximal to the splenic flexure, for example from a defunctioning colostomy site, is not suitable for determining the length of aganglionic segment. Frozen section facilities for conventional staining should therefore be available during definitive surgery for Hirschsprung's disease. The demonstration of non-specific esterase activity in ganglion cells on full thickness biopsies may also be useful preoperatively, but because this technique does not stain nerve fibres it has no advantage in identifying the transition zone.

In common with other authors' experience we have found that false negative results may occur in some situations. ${ }^{12} 13$ Firstly, there were two neonates who had negative biopsies when performed during the first week of life who subsequently had positive histochemical features of Hirschsprung's disease. The reason for this anomaly is unknown, but may be because of immaturity of the enzyme system ${ }^{12} 1415$ Secondly, there was a false negative in a patient with total colonic aganglionosis. ${ }^{12} 16$ An explanation for this false negative $\mathrm{AChE}$ has been offered on embryological 


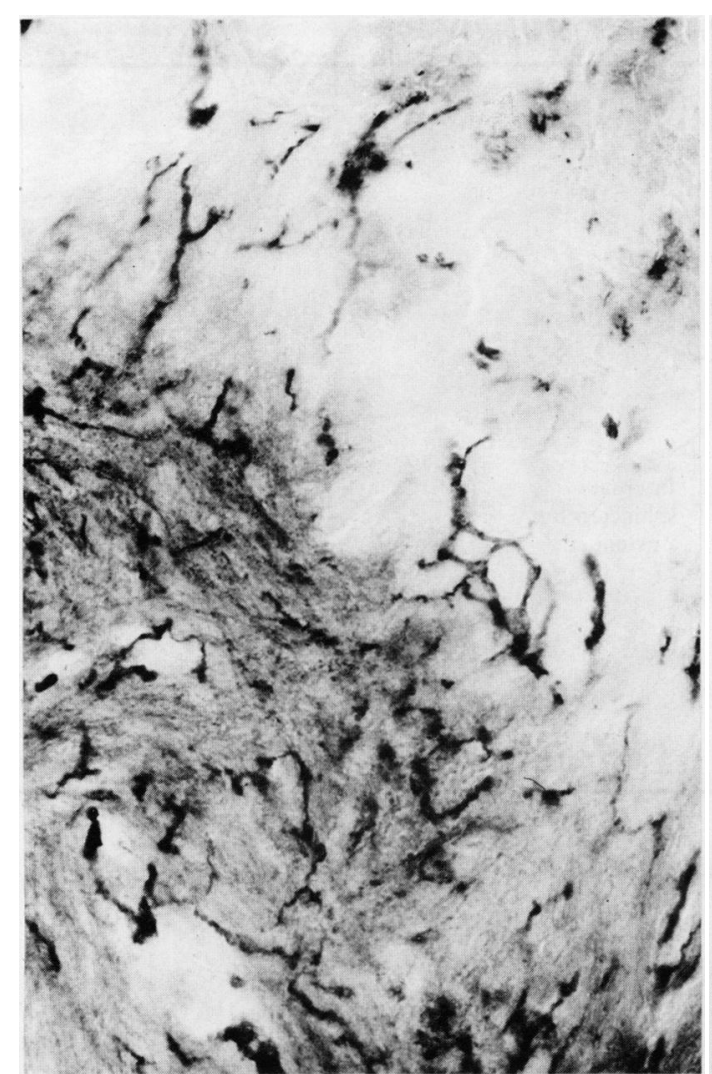

Fig.4

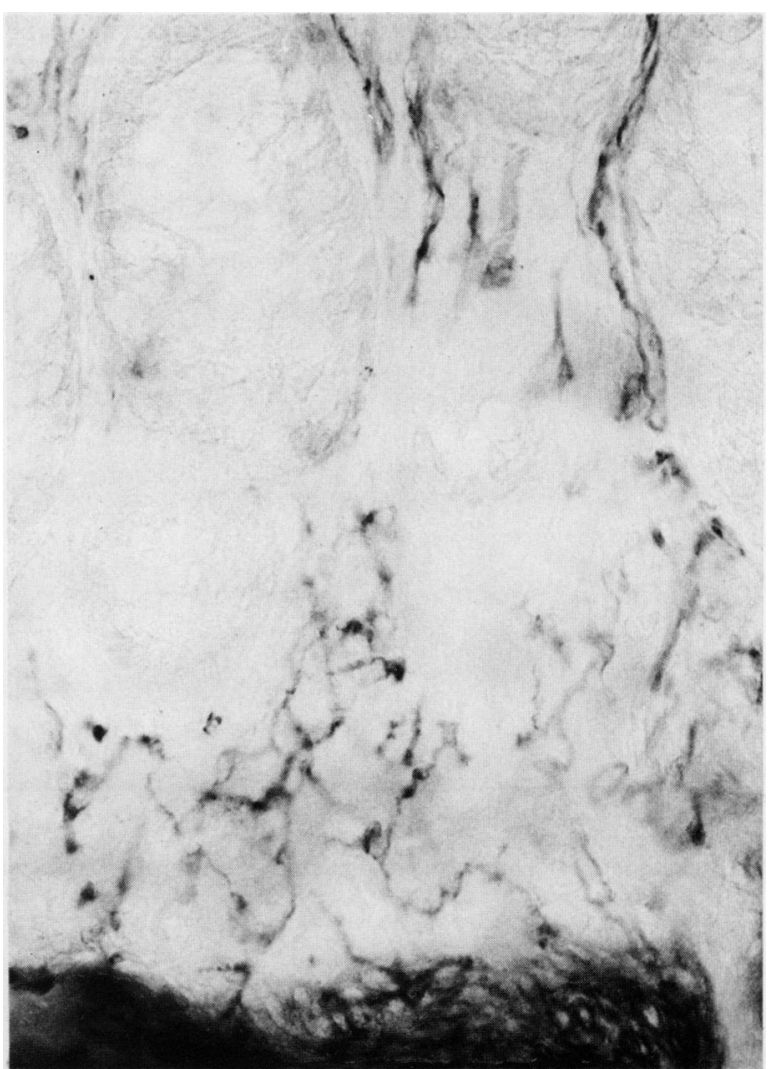

Fig. 5

Fig. 4 Atypical histochemical features. Rectal mucosa from a neonate with subacute intestinal obstruction showing atypical distribution of AChE positive nerve fibres in the muscularis mucosae and lower lamina propria. The child remains well on follow up with no surgical treatment. Another neonate with similar atypical features, in a biopsy at 4 days of age, subsequently developed the clinical and histochemical features of Hirschsprung's disease. ( $\times 500$ original magnification. AChE method).

Fig. 5 Atypical histochemical features. Rectal mucosa from a 12 year old girl with constipation, showing a network of thin $A C h E$ positive nerve fibres in the lamina propria but not in the muscularis mucoseae. A few weeks later she developed the clinical and histological features of ulcerative colitis. ( $\times 500$ original magnification. AChE method).

grounds by Okamoto and Uede. ${ }^{17}$ Thirdly, false negative results occur in patients with ultrashort segment Hirschsprung's disease, a condition which is essentially a defect of the internal anal sphincter. This condition should more appropriately be termed 'achalasia of the internal sphincter', the diagnosis depending on anorectal manometry, or biopsy of the internal sphincter muscle if sphincterotomy is performed. ${ }^{318}{ }^{19}$ False negative results may also be caused by technical faults such as a delay in freezing, and we recommend that the biopsies be processed alongside a control positive AChE specimen to minimise this possibility.
In addition to the well defined abnormalities described above, a further 19 patients were noted to have a fine network of AChE positive nerve fibres in the lamina propria and/or muscularis mucosae, but this appearance did not correlate with any pattern of clinical behaviour and is not a feature of Hirschsprung's disease. ${ }^{20}$

In conclusion, the histochemical demonstration of AChE activity in suction rectal biopsies is an accurate technique, provided that one is aware of the pitfalls outlined, and we recommend that it replace full thickness biopsy in the screening for and diagnosis of Hirschsprung's disease. Equivocal, 
Table 2 Management of patients with chronic constipation and normal histochemistry

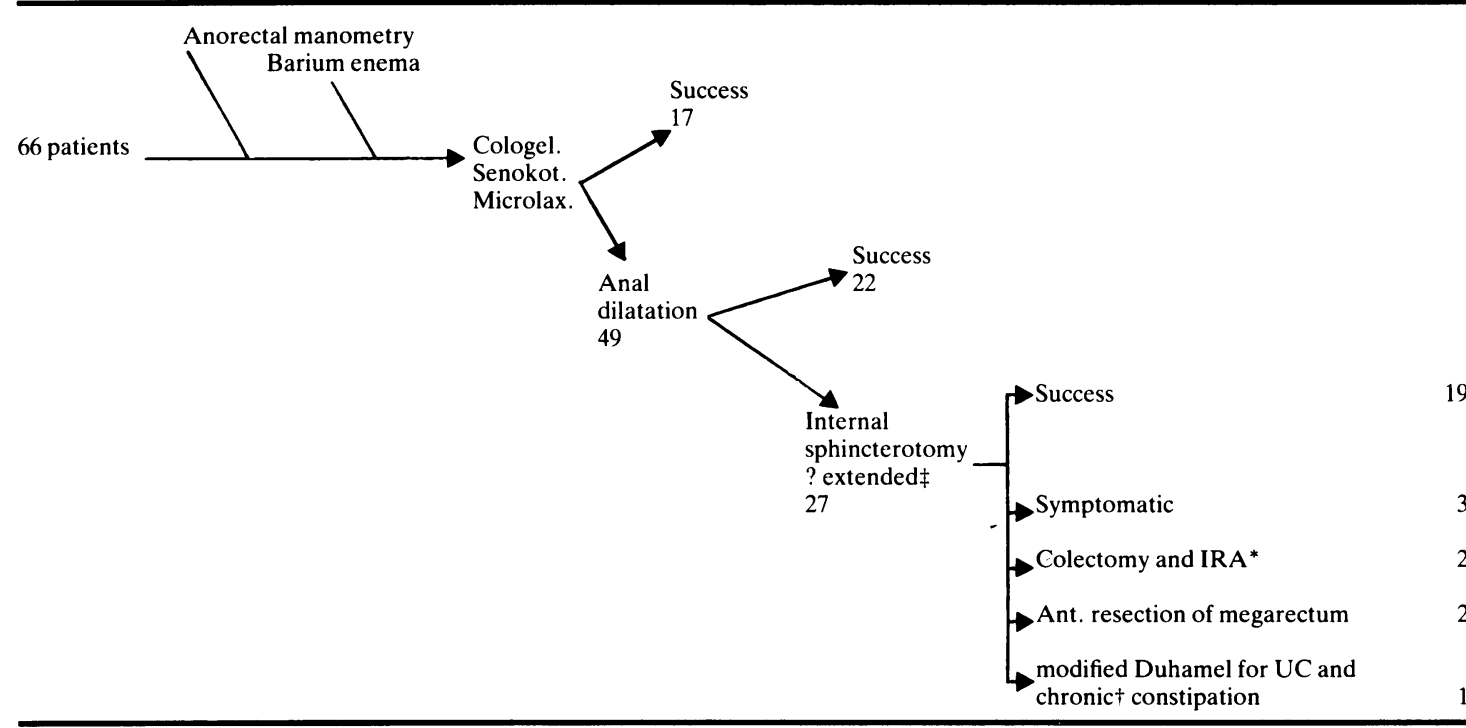

IRA ileo-rectal anastomosis

+ UC- ulcerative colitis

$\ddagger$ Extended internal sphincterotomy performed if initial partial sphincterotomy unsuccessful.

atypical, or negative results when contradicting clinical features are an indication for repeat biopsy or full thickness biopsy. In addition, the technique provides a research tool for the investigation of disorders of large bowel parasympathetic innervation and may yield further clues as to the aetiology of Hirschsprung's disease and related disorders.

\section{References}

1 Meier-Ruge W. Hirschsprung's disease: its aetiology, pathogenesis and differential diagnosis. Curr Topics Pathol 1974; 59: 131-79.

2 Aavanson I, Nixon HH. A clinical evaluation of anorectal pressure studies in the diagnosis of Hirschsprung's disease. Gut 1972; 13: 138-46.

3 Lake BD, Puri P, Nixon HH et al. Hirschsprung's disease. Arch Pathol Lab Med 1978; 102: 244-7.

4 Morkawa Y, Donahoe PK, Hendren WH. Manometry and histochemistry in the diagnosis of Hirschsprung's disease. Pediatrics 1979; 63: 865-71.

5 Scobie WG, Mackinlay GA. Anorectal myectomy in treatment of ultra-short segment Hirschsprung's disease. Arch Dis Child 1977; 52: 713-5.

6 Ravitch MM. Anal ileostomy with sphincter preservation in patients requiring total colectomy for benign conditions. Surgery 1948; 24: 170.
7 Meier-Ruge W, Lutterbuck PM, Herzog B et al. Acetylcholinesterase activity in suction biopsies of the rectum in the diagnosis of Hirschsprung's disease. $J$ Pediatr Surg 1972; 7: 11-7.

8 Chow CW, Chan WC, Yue PCK. Histochemical criteria for the diagnosis of Hirschsprung's disease. $J$ Pediatr Surg 1977; 12: 675-9.

9 Hamondi AB, Reiner CB, Boles ET et al. Acetylcholinesterase staining activity of rectal mucosa. Arch Pathol Lab Med 1982; 106: 670-2.

10 Filipe MI, Lake BD. Histochemistry in pathology. Edinburgh: Churchill Livingstone, 1983.

11 Garrett JR, Howard ER. Myenteric plexus of the hind-gut; developmental abnormalities in humans and experimental animals. In: Development of the autonomic nervous system. London: Pitman Medical, 1981: 326-43.

12 Van der Staak FHJ. Reliability of the acetylcholinesterase reaction in rectal mucosa biopsies for the diagnosis of Hirschsprung's disease. Z Kinderchiv 1981; 34: $36-43$.

13 Huntley CC, Shaffner L de S, Challa VR et al. Histochemical diagnosis of Hirschsprung's disease. Pediatrics 1982; 69: 755-61.

14 Elema JD, de Vries JA, Vos LJM. Intensity and proximal extension of acetylcholinesterase activity in the mucosa of rectosigmoid in Hirschsprung's disease. J Pediatr Surg 1973; 8: 361.

15 Almoyna CM, Claver M, Monereo J et al. [Letter.] J Pediatr Surg 1978; 13: 351-2.

16 Careskey JM, Weber TR, Grosfield JL. Total colonic 
aganglionosis. Am J Surg 1982; 143: 160-8.

17 Okamoto E, Ueda T. Embryogenesis of intramural ganglia of the gut and its relation to Hirschsprung's disease. J Pediatr Surg 1967; 2: 437.

18 Venugopal S, Mancer K, Shandling B. The validity of rectal biopsy in relation to morphology and distribution of ganglion cells. J Pediatr Surg 1981; 16: 433-7.
19 Nixon HH, Lake B. Not Hirschsprung's disease - rare conditions with some similarities. S Afr J Surg 1982; 20: 97-104.

20 Toorman J, Bots GTAM, Vio PMA. Acetylcholinesterase activity in rectal mucosa of children with obstipation. Virchows Arch [Pathol Anat] 1977; 376: 159-64. 